\title{
Kinematics of the Maneuver of the Multifunctional Machine for Metallurgical Furnaces
}

\author{
Evgeny Mityushov ${ }^{1, *}$, Egor Zinovich ${ }^{1}$, and Maxim Kosulin ${ }^{1}$ \\ ${ }^{1}$ The First President of Russia B.N. Yeltsyn Ural Federal University, Institute of New Materials and \\ Technologies, Department of mechanical engineering, Department of Metallurgical and Rotary Ma- \\ chines,620002, Mira 28, Yekaterinburg, Russia Federation.
}

\begin{abstract}
The article proposes a simulation model of a loading machine for metallurgical furnaces and builds a mathematical model that makes it possible to find optimal trajectories of motion and obtain laws of change in kinematic, dynamic and inertial characteristics for a full operation cycle. The description of the machine motion is made taking into account the nonholonomy of the imposed connections and provides for the possibility of considering arbitrary laws of motion along optimal trajectories. Compliance with optimal working conditions is also ensured by the choice of a special law of motion between the nodal points on the trajectory of movement, corresponding to the change in the direction of movement of the loading machine. Based on the research results, a design scheme and a simulation model with an analytical description of the mechanism operation, including kinematic graphs, are proposed. A simulation model of a loading machine for metallurgical furnaces and a description of its operation modes are made by methods of analytical mechanics.
\end{abstract}

\section{Introduction}

In the steelmaking industry, radical quality changes have recently taken place due to the replacement of the open-hearth method by Basic oxygen steelmaking, creation of large basic oxygen units, powerful electric arc furnaces and new installations for special metallurgy, development and wide introduction of continuous casting machines [1].

In the age of scientific and technological progress, the production of high-quality steel with high specific strength plays an especially important role. An increase in the power parameters of machines and equipment, and a decrease in their metal consumption require the production of special and highly pure steels. For the production of special steels, electric steel-making shops were built with electric furnaces with a capacity of 200 tons with comprehensive mechanization and widespread use of automation [1, p.9].

Charging machines in metallurgical production, tools for feeding, loading and unloading melting materials or ingots of various kinds of furnaces and heating wells [2].

For most multi-drive charging machines, the full working cycle is divided into separate periods during which different parts of the machine can execute commands. Additionally,

\footnotetext{
*Corresponding author: mitushov@urfu.ru
} 
the number of segments performing the movement varies at different time intervals. The working cycle of the machine is simulated with the corresponding trajectory in the configuration space. The path ends are fixed and show the start and end positions of the machine. The trajectory type is variable, but always fulfills the functional tasks of the work cycle. There are two methods of controlling the operation of the machine: sequential inclusion of its individual segments in the work and coordinated motion. The use of a coordinated motion, in which several segments simultaneously participate in separate intervals, significantly reduces the execution time of a working operation, as opposed to the sequential inclusion of individual segments. The key to improving work efficiency is the selection of suitable laws of motion of the machine segments. The mathematical basis for coordinated motion control can be the method of setting the trajectory of motion of a mechanical system with several degrees of freedom of an interpolation curve in the corresponding configurational space, passing through the interpolation nodes, determined by the operating conditions of a particular machine.

An illustrative example of a floor filling machine operating at the German plant Karl Diederichs $\mathrm{GmbH} \& \mathrm{Co}$. KG, can be viewed by following the link of the reference [3].

The goal of the study is to find the optimal motion trajectories of the floor loading, taking into account the nonholonomy of the applied connections, to build a mathematical simulation model of the machine's motion with the possibility to consider different laws of motion along optimal trajectories and to construct cycle schemes of the kinematic characteristics of its motion.

\section{Analysis of the MM3-3M charging machine}

The methods of analytical mechanics are used to construct a simulation model of the loading machine for metallurgical furnaces and describe the modes of its operation. A mathematical simulation was carried out to describe the full working cycle of the movement of the loading machine of the MMZ-3M multifunctional metallurgical furnace charging machine.

The multifunctional charging machine is a three-wheeled cart with one rear driving steering wheel carrying an arm in front of it. The MMZ-3M machine is self-propelled with an electric drive (Fig. 1). It is operated in conditions of above- and below-zero temperatures and is intended for work in electric steel-making shops of metallurgical production sites.

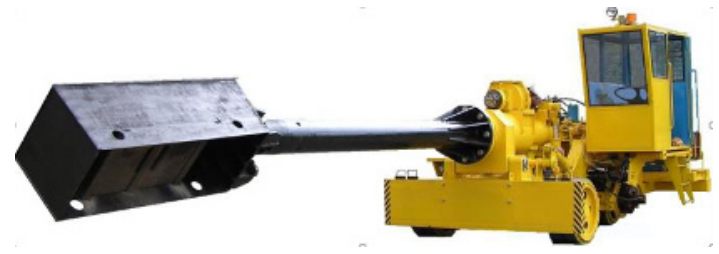

Fig. 1. Charging machine MM3-3M.

The main purpose of the machine: transportation of molds with ferroalloys for their installation in a heating furnace, as well as transportation of molds with heated ferroalloys for loading them into an electric arc furnace.

The operation of the MMZ-3M charging machine is shown in the video [4].

Based on the regulated requirements for the optimal conditions of the loading process and the working site, the route of the working stroke of the machine is selected, consisting of six phases of movement:

1. All segments of the system make a translational motion when leaving the place of receiving the cargo at the distance necessary for the subsequent maneuver with a turn. 
2. All segments make a plane motion when leaving the place of receiving the load, while the center point of the front wheel axle moves along a circle arc.

3. The machine moves forward along the furnace line.

4. All segments make a plane motion towards the furnace. The center point of the front wheel axle moves in a circular arc.

5. All segments make a forward movement towards the furnace. The furnace is being loaded.

\section{Simulation of the optimal trajectory of the machine in the space of the EAF-100 electric arc furnace shop}

To simulate the movement of the charging machine, we chose the plan of the EAF-100 electric steel-making shop of length L1 and width L2, with which the Oxy coordinate system is associated (the Ox axis is directed along the shop).

Taking into account the regulated phases of movement of the charging machine, when constructing the optimal trajectory of its movement, the following model parameters are introduced: $(\mathrm{x} 1, \mathrm{y} 1)$ - coordinates of the point of the beginning of the turn of the charging machine on the shop floor plan, $(\mathrm{x} 1, \mathrm{y} 1)$ - coordinates of the point of the end of the turn of the charging machine, $\mathrm{r} 0$ is the minimum possible turning radius of the charging machine, $\mathrm{r} 1$ is the radius of the trajectory of the midpoint of the front wheel

axle when leaving the place of receiving the load, $r 2$ is the radius of the trajectory of the midpoint of the axle of the front wheels when approaching the furnace from the place of receiving the load, 1 is distance traveled by the machine along the furnace line.

Depending on the relative position of the place of receiving the cargo and the furnace, two options for constructing the optimal trajectory are possible.

1. The condition is met: $x_{2}-x_{1} \leq r_{2}-r_{1}\left(r_{1}>r_{0}, r_{2}>r_{0}\right) \mathrm{x}$

The trajectory of the front wheel axle midpoint is piecewise-continuous and consists of two quarters of circles with radii $\mathrm{r} 1$ and $\mathrm{r} 2$.

2. The condition is met: $x_{2}-x_{1}>r_{2}-r_{1}\left(r_{1}>r_{0}, r_{2}>r_{0}\right)$

The trajectory of the midpoint of the front wheel axle is piecewise continuous and consists of two quarters of circles with radii r1 and r2, separated by a straight-line segment of length 1 parallel to the furnace line.

In the first case, the trajectory of the midpoint of the front wheel axle is piecewise continuous and consists of two quarters of circles with radii $\mathrm{r} 1$ and $\mathrm{r} 2$. The trajectory equations in parametric form are:

$$
\begin{gathered}
\left\{\begin{array}{l}
x_{C}=x_{1}-r_{1} \cos u, \quad 0 \leq u \leq \frac{\pi}{4}, \\
y_{C}=y_{1}+r_{1} \sin u, \quad 0
\end{array}\right. \\
\left\{\begin{array}{c}
x_{C}=x_{2}-\left(r_{2}-r_{2} \sin u\right), 0 \leq u \leq \frac{\pi}{4} \\
y_{C}=y_{2}-r_{2} \cos u,
\end{array}\right.
\end{gathered}
$$

The radii $\mathrm{r} 1$ and $\mathrm{r} 2$ of the trajectories in the first case are determined from the system of equations:

in which

$$
\left\{\begin{array}{c}
r_{1}+r_{2}=L_{2} \\
r_{2}-r_{1}=\left|x_{2}-x_{1}\right|
\end{array}\right.
$$

$$
r_{1}=\frac{1}{2}\left(L_{2}-\left|x_{2}-x_{1}\right|\right), \quad r_{2}=\frac{1}{2}\left(L_{2}+\left|x_{2}-x_{1}\right|\right) .
$$

In the second case, the optimal trajectory is sought from the condition of the minimum total distance 


$$
s=\frac{\pi r_{1}}{2}+l+\frac{\pi r_{2}}{2}
$$

using the linear programming task under the conditions

Here we have two solutions

$$
r_{1}>r_{0}, r_{2}>r_{0}, r_{1}+r_{2}=L_{2} \text {. }
$$

and

$$
l=\left|x_{2}-x_{1}\right|+L_{2}+2 r_{0}, \quad r_{1}=r_{0}, \quad r_{2}=L_{2}-r_{0}
$$

$$
l=\left|x_{2}-x_{1}\right|+L_{2}+2 r_{0}, r_{1}=L_{2}-r_{0}, r_{2}=r_{0} \text {. }
$$

\section{Equations of motion of the charging machine}

The charging machine moves along the floor, which is presented as a non-holonomic connection. Its position is determined by three generalized coordinates: $x(t), y(t)$ are the coordinates of the midpoint of the front wheel axle and $\varphi(t)$ is the angle of rotation of the machine

To simulate the movement of the charging machine along the found optimal trajectories, the parameter $\mathrm{u}$ included in equations (1) and (2) is presented as a function of time. In this case, we will consider two cases of motion - uniform movement and movement in the mode of smooth start-off of the charging machine and its smooth braking.

In the first case

$u_{1}(t)=\frac{v_{1}}{r_{1}} t, 0 \leq t \leq T_{1} ; \quad u_{2}(t)=\frac{v_{2}}{r_{2}} t, 1 \leq t \leq T_{2}$,

$v$ - is the constant speed of the center axle point of the front wheels.

In the second case, the law of soft start and braking is used.

$$
\begin{aligned}
& u_{1}(t)=\frac{\pi}{2}\left[6\left(\frac{t}{T_{1}}\right)^{5}-15\left(\frac{t}{T_{1}}\right)^{4}+10\left(\frac{t}{T_{1}}\right)^{3}\right] 0 \leq t \leq T_{1}, \\
& u_{2}(t)=\frac{\pi}{2}\left[6\left(\frac{t}{T_{2}}\right)^{5}-15\left(\frac{t}{T_{2}}\right)^{4}+10\left(\frac{t}{T_{2}}\right)^{3}\right] T_{1} \leq t \leq T, .
\end{aligned}
$$

The functions $u u 1(t)$ and $u 2(t)$ are identical to the generalized coordinate $\varphi(t)$ and set the rotation of the charging machine around the center point of the front wheel axle. In this case, the position of an arbitrary point $M$ of the machine, given in the moving coordinate system $C X Y$, is determined using the Rodrigues formula from the equality:

$$
\left(\begin{array}{c}
x(t) \\
y(t) \\
0
\end{array}\right)=\left(\begin{array}{c}
x_{C}(t)+X_{M} \\
y_{C}(t)+Y_{M} \\
0
\end{array}\right)+\left[R^{2}(1-\cos u(t))+R \sin u(t)\right]\left(\begin{array}{c}
X_{M} \\
Y_{M} \\
0
\end{array}\right)
$$

in which $R=\left(\begin{array}{ccc}0 & -e_{z} & e_{y} \\ e_{z} & 0 & -e_{x} \\ -e_{y} & e_{x} & 0\end{array}\right)$ - pivot matrix for the unit vector of

$\vec{e}=\{0,0,1\}$

\section{Movement of the charging machine in the mode of soft start and brakin}

As an example, we consider the movement of the charging machine in the mode of soft start and braking with the following parameters: $L_{1}=34,5 \mathrm{~m}, L_{2}=25 \mathrm{~m}, x_{1}=16,6 \mathrm{~m}$, $y_{1}=0, x_{2}=22,6 \mathrm{~m}, y_{2}=25 \mathrm{~m}, T_{1}=5 \mathrm{sec} ., T_{2}=10 \mathrm{sec}$. 
The animation frames for the movement of the axle of the front wheels of the multifiller machine are shown in figure 2.

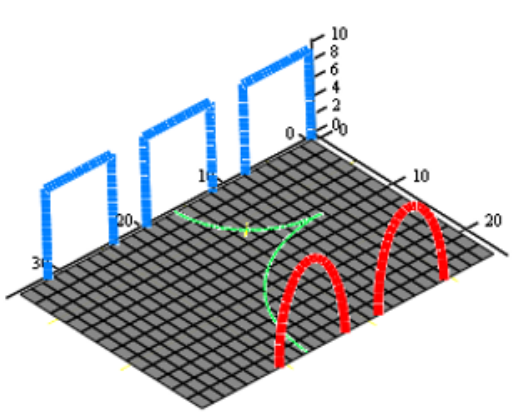

$$
t=2,5 s \text {. }
$$

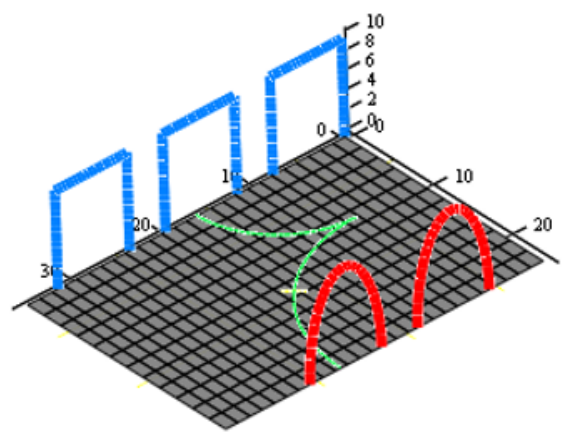

$t=10 \mathrm{~s}$.

Fig. 2. Animation frames of the axle motion of the front wheels of the charging machine.

The laws of motion obtained using a mathematical simulation model and represented by equations (3) - (5), allow one to build graphs of changes in generalized coordinates. The corresponding cycle schemes of the generalized coordinates are presented in figure 3.
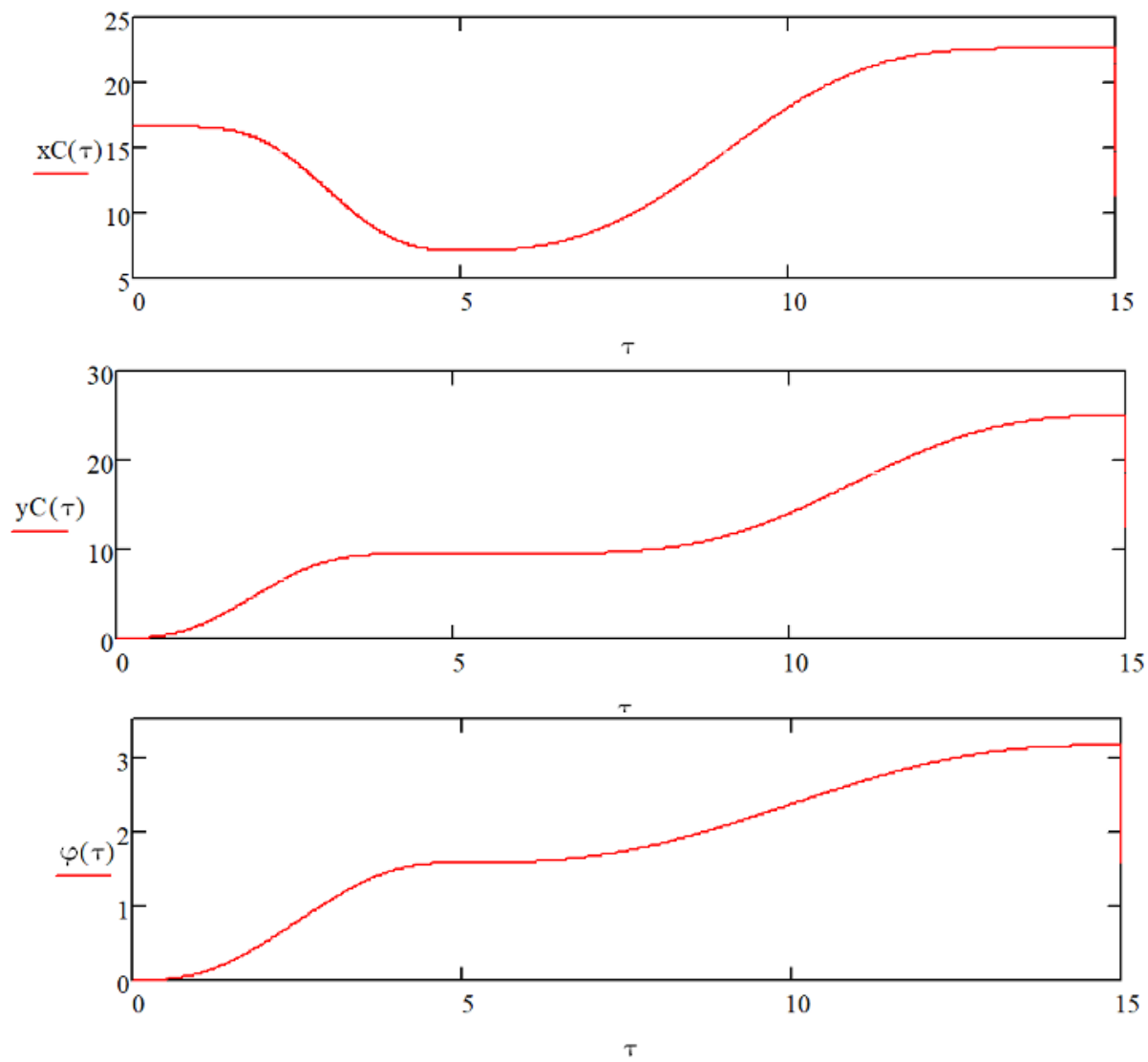

Fig. 3. Cycle schemes of changes in generalized coordinates. 
By differentiating equalities (3) - (5) with consideration of time, the cycle schemes of the generalized speeds in the working cycle of the charging machine are found. The corresponding cycle schemes of generalized speeds are presented in figure 4.
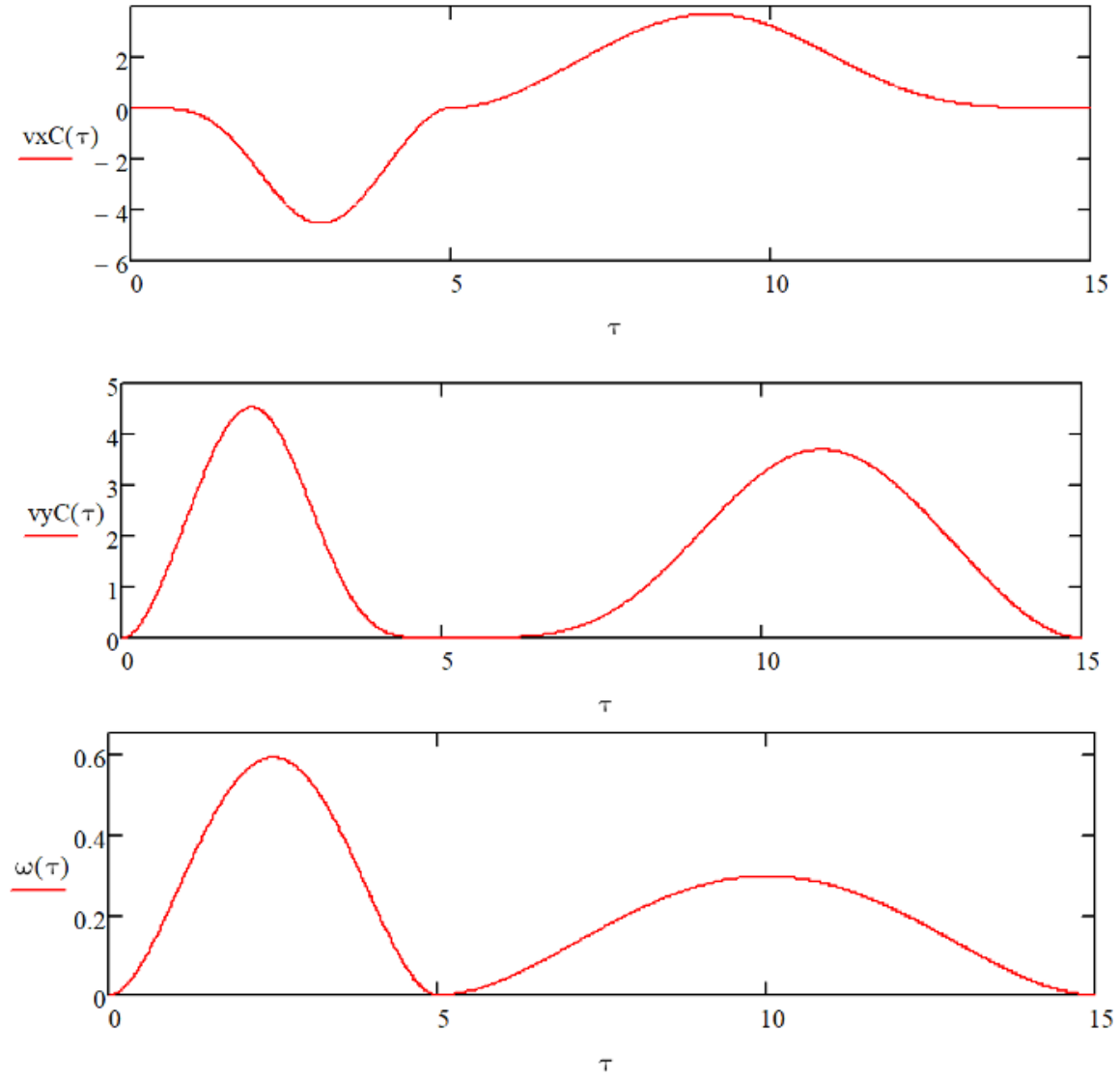

Fig. 4. Cycle schemes of changes in generalized speeds

\section{Conclusion}

The article presents a mathematical simulation of the maneuver in the working cycle of the MMZ-3M charging machine, using of analytical mechanics and numerical implementation in the Mathcad computer algebra package. We've some variants of the optimal movements of the machine in the space of the DSP-100 workshop. Cycle schemes of generalized coordinates defining the position of the machine and generalized speeds are constructed. The resulting kinematic analysis makes it possible to perform further dynamic analysis of the operation of the charging machine with the determination and optimization of energy costs in the working cycle of its operation. Maneuvering is versatile and can be designed and applied in other locations and purposes.

\section{References}

1. A. I. Tselikov, P. I. Polukhin, V. M. Grebenik, F. K. Ivanchenko, M. A. Tylkin, A. A. Korolev, V. P. Lopukhin, D. A. Storozhik, B. A. Pavlenko, A. A. Tselikov, I. M. Elin- 
son, V. I. Zyuzin Machines and units of metallurgical plants, Moscow «Metallurgy» (1988) p. 9-12

2. Loading machines in metallurgical production (Electronic Materials) URL: https://azbukametalla.ru/entsiklopediya/z/zagruzochnye-mashiny.html (24.02.2021)

3. American type charging machine movement (Electronic Materials) URL: https://www.youtube.com/watch?v=9ex7zrKaRwQ (27.02.2021)

4. Charging machine MMZ-3M operation (Electronic Materials) URL: https://www.youtube.com/watch?v=3HWPvwyR_wg (27.02.2021) 